Case Report

\title{
Loss of HER2 Positivity after Trastuzumab in HER2-Positive Gastric Cancer: Is Change in HER2 Status Significantly Frequent?
}

\author{
Yu Ishimine, ${ }^{1}$ Akira Goto, ${ }^{1}$ Yoshito Watanabe, ${ }^{2}$ Hidetaka Yajima, ${ }^{3}$ Suguru Nakagaki, \\ Takashi Yabana, ${ }^{1}$ Takeya Adachi, ${ }^{1}$ Yoshihiro Kondo, ${ }^{1}$ and Kiyoshi Kasai ${ }^{4}$ \\ ${ }^{1}$ Department of Gastroenterology, Otaru General Hospital, 1-2-1 Wakamatu, Otaru 047-8550, Japan \\ ${ }^{2}$ Department of Surgery, Otaru General Hospital, 1-2-1 Wakamatu, Otaru 047-8550, Japan \\ ${ }^{3}$ Department of Gastroenterology, Rheumatology, and Clinical Immunology, Sapporo Medical University, S1W17, Chuo-ku, \\ Sapporo 060-8556, Japan \\ ${ }^{4}$ Department of Laboratory Medicine, Otaru General Hospital, 1-2-1 Wakamatu, Otaru 047-8550, Japan
}

Correspondence should be addressed to Akira Goto; goa@sapmed.ac.jp

Received 22 October 2014; Accepted 10 March 2015

Academic Editor: Olga I. Giouleme

Copyright (C) 2015 Yu Ishimine et al. This is an open access article distributed under the Creative Commons Attribution License, which permits unrestricted use, distribution, and reproduction in any medium, provided the original work is properly cited.

\begin{abstract}
Trastuzumab has recently been introduced as a treatment for HER2-positive metastatic and/or unresectable gastric cancer (MUGC); however, compared with breast cancer, some issues concerning HER2 and trastuzumab therapy for gastric cancer remain unclear. A 74-year-old woman received trastuzumab-containing chemotherapy for HER2-positive MUGC. She had a marked response to 8 months of chemotherapy, and gastrectomy and hepatic metastasectomy with curative intent were performed. The resected specimen showed complete loss of HER2 positivity in the residual tumor. For MUGC, a change in HER2 status during the course of the disease with or without chemotherapy has rarely been reported. However, in breast cancer, a significant frequency of change in HER 2 status during the course of disease has been reported, and reevaluation of HER2 positivity in metastatic/recurrent sites is recommended. The choice of trastuzumab for MUGC is currently based on the HER2 status of the primary tumor at the time of initial diagnosis, without reassessment of HER2 status during the course of disease and/or in metastatic/recurrent sites, on the assumption that HER2 status is stable. However, our case casts doubt on the stability of HER2 in gastric cancer.
\end{abstract}

\section{Introduction}

The primary treatment for patients with metastatic and/or unresectable gastric cancer (MUGC) is systematic chemotherapy. Although the combination of 5-fluorouracil and cisplatin has been the standard regimen for the treatment of MUGC, new cytotoxic agents such as S-1, capecitabine, paclitaxel, docetaxel, oxaliplatin, irinotecan, and their combinations have proven to be more effective and better tolerated, resulting in the improvement of median survival up to 13 months [1-3]. In addition, recent research has shown that adding trastuzumab, a humanized monoclonal antibody targeting the human epidermal growth factor receptor 2 (HER2) protein, to chemotherapy (fluoropyrimidines and cisplatin) for patients with MUGC with HER2 overexpression and/or gene amplification (the so-called HER2-positive gastric cancer) has a significant superiority to chemotherapy alone in terms of overall survival (median survival 13.8 months with trastuzumab versus 11.1 months with chemotherapy alone) [4].

We report a case in which chemotherapy containing trastuzumab made it possible to perform curative gastrectomy in a patient with HER2-positive MUGC at initial diagnosis; this case is noteworthy in that HER2 positivity in resected specimens of the residual tumor was completely lost. Loss of HER2 positivity after treatment with trastuzumab for HER2-positive MUGC has rarely been reported. HER2 status is an essential biomarker for the effectiveness of trastuzumab, and consequently, knowledge of HER2 stability during the course of disease is critical for determining the introduction, the continued use, or elimination of trastuzumab. We discuss 


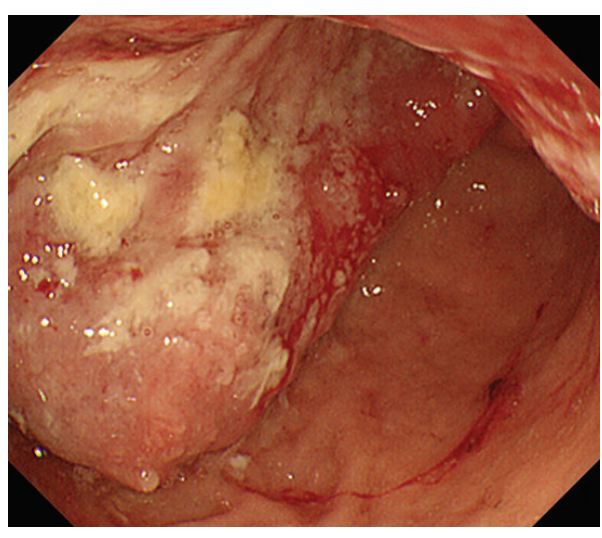

(a)

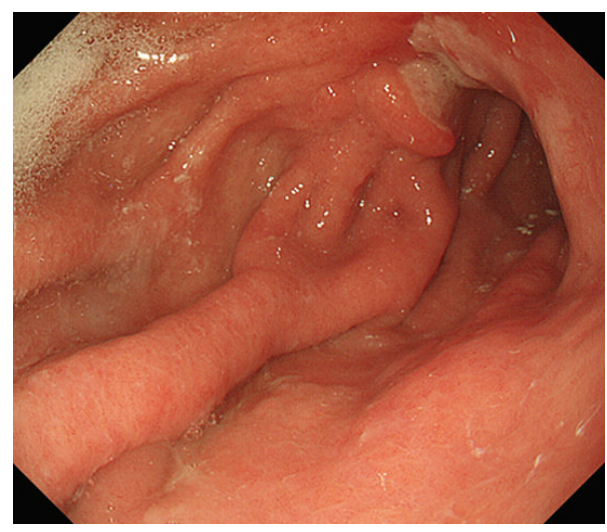

(b)

FIGURE 1: Endoscopic findings. (a) Gastroscopy at presentation revealed an ulcerative mass with raised margins along the lesser curvature extending from the lower body of the stomach to the antrum. (b) After completion of the 11th course of chemotherapy (capecitabine + CDDP + trastuzumab), the gastric tumor is decreased remarkably.

the stability of HER2 status in gastric cancer and provide a literature review of this important topic.

\section{Case Report}

A 74-year-old woman with the chief complaint of palpable masses in her abdomen presented to our hospital in May 2013. Physical examination revealed one $50-\mathrm{mm}$ mass in the upper central abdomen and three 20-30-mm palpable masses in the other parts of her abdomen. Esophagogastroduodenoscopy (EGD) revealed an ulcerative mass with raised margins along the lesser curvature extending from the lower body of the stomach to the antrum (Figure 1(a)). Biopsy of the lesion revealed a well-differentiated tubular adenocarcinoma (Figure 2(a)), and HER2 immunohistochemistry (IHC) scoring of the tumor in the biopsy specimen was $3+$, indicating HER2-positivity (Figure 2(b)). Contrast-enhanced computed tomography (CT) demonstrated marked wall thickening of the lower part of the stomach with irregular outer borders and an increased density of the surrounding fat tissue, forming a mass that was $80 \times 45 \mathrm{~mm}$ (Figure 3(a)). Several nodules in the liver were detected. Two of these nodules in the lateral segment of the liver were directly contiguous from the gastric mass and were considered direct invasion of the gastric cancer (Figure 3(b)). Invasion into the pancreas was suggested by obliteration of the fat plane between the gastric mass and the pancreas. CT also revealed multiple nodules in the peritoneal cavity, a few masses in the abdominal wall, and ascites. Many perigastric lymph nodes and several para-aortic lymph nodes were swollen.

With this information, this patient's gastric cancer was classified as stage IV (cT4bN3aM1) according to the 7th TNM classification of the American Joint Committee on Cancer/Union for International Cancer Control (AJCC/UICC) staging system for gastric cancer. She was treated with 3week courses of capecitabine $\left(2000 \mathrm{mg} / \mathrm{m}^{2} /\right.$ day $)$ orally on days $1-14$, cisplatin $\left(80 \mathrm{mg} / \mathrm{m}^{2}\right)$ intravenously on day 1 , and trastuzumab ( $8 \mathrm{mg} / \mathrm{kg}$ loading dose followed by $6 \mathrm{mg} / \mathrm{kg}$ ) intravenously on day 1 . From the 3 rd course, the doses of capecitabine and cisplatin were reduced to $1500 \mathrm{mg} / \mathrm{m}^{2} /$ day and $60 \mathrm{mg} / \mathrm{m}^{2}$, respectively, due to nonhematologic toxicity (nausea, anorexia, and renal impairment). After completion of the 4 th course of chemotherapy, at 3 months after diagnosis, a $76 \%$ decrease in measureable tumor burden compared to baseline according to RECIST 1.1 criteria was observed. From the 6 th course, cisplatin was discontinued due to renal impairment, but treatment with capecitabine and trastuzumab continued. After completion of the 11th course, at 8 months after diagnosis, EGD showed a marked reduction of the gastric tumor (Figure $1(\mathrm{~b})$ ), and contrastenhanced CT (Figures 3(c) and 3(d)) showed a 90\% reduction in measureable tumor burden. No metastases were detected in the liver except for one small nodule in the lateral segment (Figure 3(d)). Ascites and metastases in the peritoneal cavity, abdominal wall, and para-aortic lymph nodes were completely resolved.

Given this response to the treatment, complete resection of the residual tumor was deemed possible, and 9 months after the diagnosis, distal gastrectomy with D2 lymphadenectomy and hepatic metastasectomy of the small nodule in the lateral segment were performed. Operative findings showed no macroscopic peritoneal metastasis and the absence of peritoneal lavage cytology. Macroscopic complete resection was possible. Pathologic findings of the $36 \times 25 \mathrm{~mm}$ mass from the resected stomach revealed a moderately differentiated adenocarcinoma with subserosal invasion (Figure 2(c)) and hyalinization and fibrosis adjacent to the tumor in the lower third of the gastric wall. Eight regional lymph nodes were positive for tumor cells. Specimens of the nodule from the resected liver revealed only hyalinization and fibrosis without tumor cells. HER2 by IHC was completely negative in the residual tumor from the resected stomach (Figure 2(d)) and lymph nodes. HER2 also was negative by fluorescence in situ hybridization (FISH). The patient received adjuvant chemotherapy comprising capecitabine and trastuzumab for 6 months, and at the completion of adjuvant chemotherapy, the patient had no signs of recurrence. 


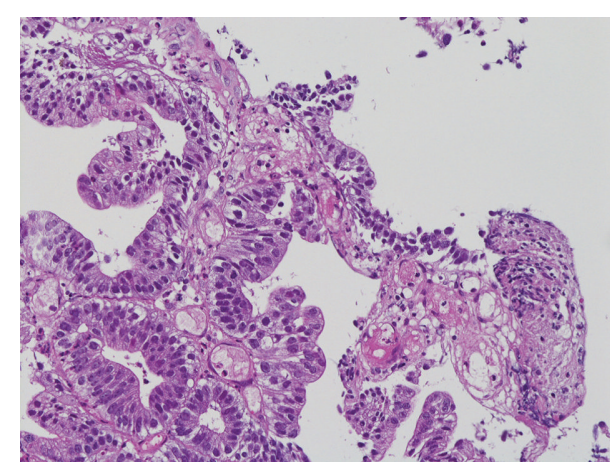

(a)

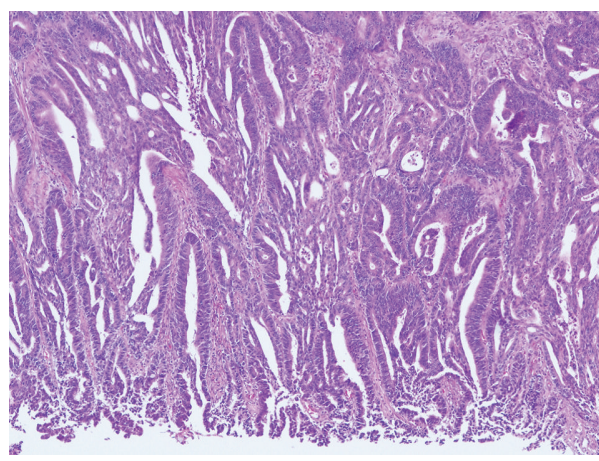

(c)

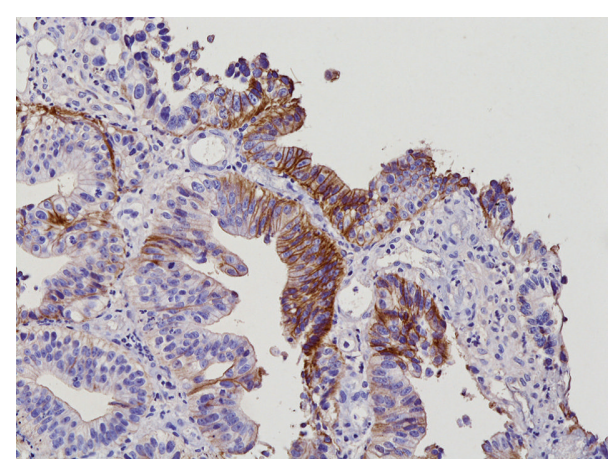

(b)

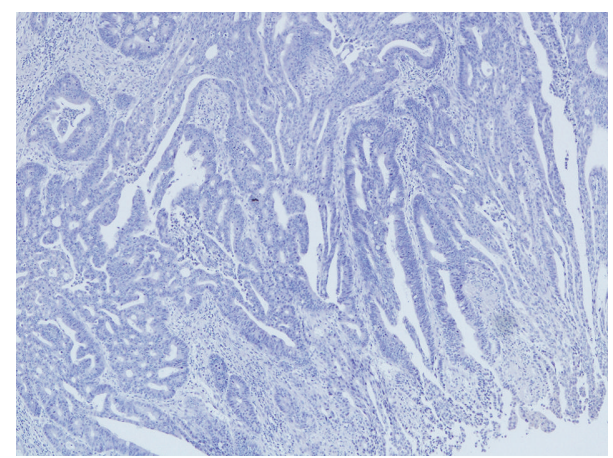

(d)

FIGURE 2: Microscopic findings of gastric cancer. (a) and (b) show biopsy specimens at presentation. (a) Histological findings show a well-differentiated tubular adenocarcinoma (hematoxylin and eosin staining, original magnification $\times 40$ ). (b) Immunohistological findings of HER2 immunostaining show strong basolateral membrane reactivity with heterogeneity. The gastric cancer is scored as 3+ by HER2 immunohistochemistry scoring criteria (original magnification $\times 40$ ). (c) and (d) show the resected specimen after chemotherapy including trastuzumab. (c) Histological findings show a moderately differentiated tubular adenocarcinoma invading into the subserosa (hematoxylin and eosin staining, original magnification $\times 40$ ). (d) Immunohistological findings of HER2 immunostaining showed no membranous reactivity in any tumor cells (original magnification $\times 40$ ).

\section{Discussion}

Approximately 15-20\% of invasive breast cancers have HER2 overexpression and/or gene amplification [5, 6]. HER2 has been used as a molecular target for breast cancer for many years. Trastuzumab was initially approved for the treatment of patients with HER2-positive metastatic breast cancer [7]. Trastuzumab is now the standard treatment in adjuvant therapy for HER2-positive early-stage breast cancer [8] and is also recommended as part of neoadjuvant chemotherapy (NAC) [9].

In breast cancer, discordance in HER2 status between the primary tumor and the metastasis/recurrence and HER2 stability during the course of disease has been evaluated in several reports. The discordance rate of HER2 status in breast cancer has been reported to be 3-16\% [10-13], with $12-19 \%$ of HER2-positive tumors in the primary site converting to HER2-negative status at the sites of metastasis/recurrence [11, 12] and 7-10\% of HER2-negative tumors converting to HER2positive status $[11,12,14]$. Conversion of the HER2 status from positive to negative may be more frequent than conversion from negative to positive. The cause of discordance of HER2 status has been attributed to changes in the molecular profile during the course of tumor progression, the differing effects of prior treatment on clonal subsets, heterogeneity of HER2 expression within the tumor, and technical errors in tissue processing and evaluation.

Several previous reports have evaluated how chemotherapy with or without anti-HER2 agents, including trastuzumab, influences HER2 expression. The discordance rate of HER2 status before and after NAC has been reported to be $0-9.5 \%$ [15-17]. In HER2-positive breast cancer, loss of HER2 positivity after NAC has been reported to be $8-32 \%$ [15, 17-19], and in HER2-negative breast cancer, gain of HER2 positivity after NAC has been reported in $0-5 \%$ of cases $[15,17]$. Niikura et al. reported that the conversion rate from positive in the primary tumors to negative in the sites of metastases was significantly higher in patients with prior chemotherapy than in patients without prior chemotherapy (17\% versus 10\%) [20]. These previous reports have suggested that chemotherapy can be associated with a change of HER2 status in breast cancer.

In addition to studying the association between HER2 status and traditional chemotherapeutic agents, several reports have analyzed the association between change in HER2 status and trastuzumab therapy. A loss of HER2 positivity in patients treated with trastuzumab therapy has been reported in $14.7-37 \%$ of HER2-positive breast cancers [14, 18-21]. 


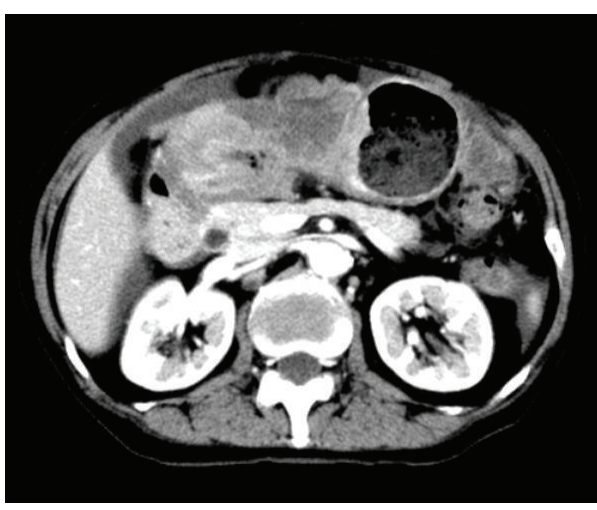

(a)

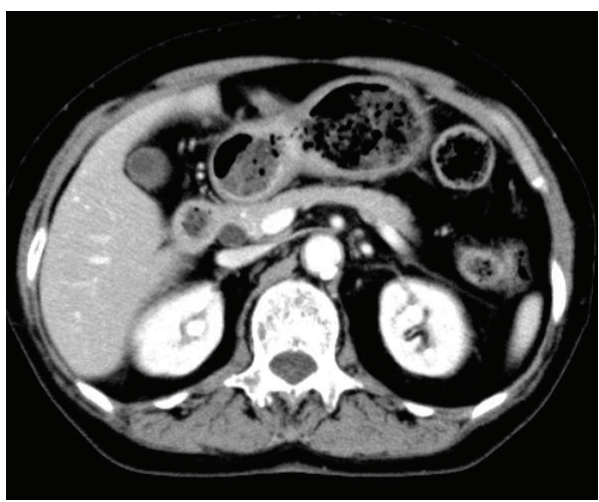

(c)

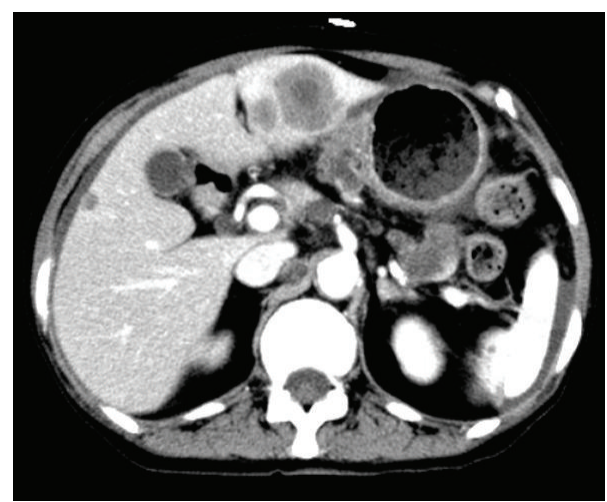

(b)

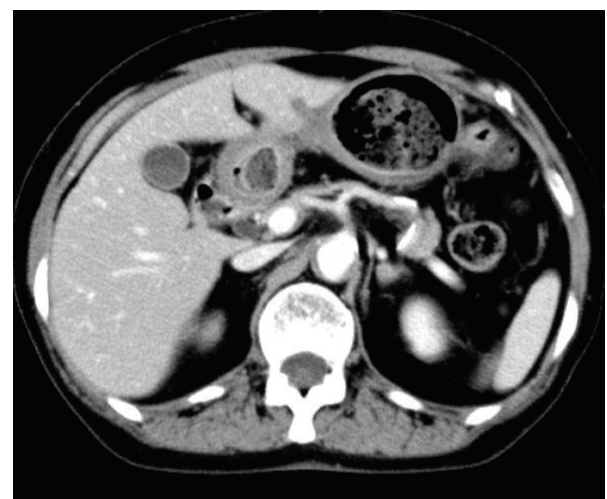

(d)

FIGURE 3: Contrast-enhanced computed tomography (CT) findings. (a) and (b) show the CT at presentation. (a) The wall of the lower part of the stomach is markedly thickened, and the density of the surrounding fat tissue is increased, forming an $80 \times 45$ mm mass. (b) Two nodules are detected in the lateral segment of the liver, and these are directly contiguous from the gastric mass. (c) and (d) show the CT findings after completion of the 11th course of chemotherapy (capecitabine + CDDP + trastuzumab). (c) The gastric mass is markedly decreased. (d) Only a small nodule in the lateral segment of the liver is detected.

Nakamura et al. reported that, in HER2-positive primary breast cancer, the rate of HER2 loss in the site of recurrence was significantly higher in patients treated with trastuzumab than in patients treated without trastuzumab $(37 \%$ versus $6 \%)$ [11]. However, others have reported that trastuzumab therapy was not significantly associated with loss of HER2 positivity in HER2-positive primary breast cancer $(13.2 \%$ with trastuzumab versus $17.9 \%$ without trastuzumab and $20 \%$ with trastuzumab versus $26 \%$ without trastuzumab) $[14,20]$. In fact, some research has shown that treatment with trastuzumab is associated with a lower rate of HER2 status conversion. Guarneri et al. reported that in HER2positive breast cancer the rate of loss of HER2 after NAC with trastuzumab was significantly lower than the rate of conversion after treatment with NAC without trastuzumab (14.7\% versus $40 \%$ ) [19]. It is evident that available results on the association between loss of HER2 status and trastuzumab therapy are conflicting. These conflicting results imply that loss of HER2 in HER2-positive breast cancer after trastuzumab therapy might not simply be caused by survival of trastuzumab-resistant clones and elimination of HER2positive clones.

HER2 overexpression and/or amplification occurs in 7$34 \%$ of gastric cancer cases $[22,23]$. In gastric cancer, the rate of HER2 status discordance between primary tumors and simultaneous metastasis in the liver or lymph nodes has been reported to be $0-12.5 \%$ [24-26]. However, HER2 stability during disease progression with or without chemotherapy has rarely been reported. In fact, our literature review identified only one case report similar to ours in which HER2 positivity in the resected specimen from curative surgery was lost after 12 courses of capecitabine + cisplatin + trastuzumab in a patient with HER2-positive MUGC [27]. However, the actual frequency of HER2 loss in HER2-positive MUGC remains unknown, because in MGC, reevaluation of HER2 status in primary sites and/or sites of metastases/recurrence during the course of disease is not typically performed.

When analyzing loss of HER2 in a sample obtained after treatment, we must consider the possibility of a false-negative result for HER2 expression on immunohistochemistry. A sample taken by biopsy is only part of a tumor, and a resected sample might be one of many tumors within the body; therefore, loss of HER2 might be a false-negative due to heterogeneity within the tumor or discordance between the sites of tumors. In fact, it is known that the heterogeneity of HER2 expression within gastric tumors is much more frequent than in breast cancer $[22,23]$. Therefore, heterogeneity might account for a small fraction of HER2 conversion. Resolution 
of this issue is quite challenging, because it is difficult in practice to obtain adequate specimens to overcome the heterogeneity of HER2 within a given tumor and to evaluate the HER2 status of every tumor within the entire body. However, we believe that our particular case was not due to a false-negative result of HER2, because we carefully evaluated HER2 status in the entire residual tumor of the resected stomach and lymph nodes. Although previous studies have demonstrated that $0-10 \%$ of cases negative for HER 2 by IHC were positive by FISH [24,25,28], we confirmed negativity of HER2 in this case by FISH as well.

Current practice guideline for the treatment of breast cancer recommended that biomarkers, including HER2 status and hormone receptors, should be evaluated not only for the primary tumor but also for the initial metastatic or recurrence tumor tissues, especially if the first determination of HER2 status is negative, in order not to lose the chance of anti-HER2 therapy [29]. Currently, the decision to use trastuzumab to treat patients with MUGC is based on the HER2 status in the primary tumor at the time of initial diagnosis without reassessment of HER2 status in primary sites and/or metastases/recurrence during the course of disease, on the assumption that HER2 status is stable. However, just as it is known that HER2 is not necessarily stable in breast cancer, our case casts some doubt on the notion of HER2 stability in gastric cancer.

Although the use of trastuzumab has been shown to be highly effective in treating HER2-positive MUCG, several issues regarding HER2 stability and trastuzumab therapy for gastric cancer remain to be elucidated. First, clinicians need to know how often HER2 status converts with tumor progression, metastasis, or treatment. If a change in HER2 status is found to be significantly frequent in gastric cancer, management strategy recommendations similar to those of breast cancer should be introduced for gastric cancer, although it will be important to consider that biopsy from metastases/recurrence or metastasectomy is invasive and burdensome for patients. If the factors that contribute to HER2 change are identified, only those patients who have such factors, rather than all patients with MUGC, would need to submit to biopsy from metastases/recurrence or metastasectomy. Second, clinicians need to evaluate whether loss of HER2 positivity in specimens obtained during the disease course in HER2-positive gastric cancer always indicates resistance to trastuzumab. As we have mentioned, HER2 status taken from samples may not always represent all tumors in the body. In the present case, although macroscopic complete resection was possible, there was still a sufficient possibility of remaining micrometastases. We decided to treat the patient with adjuvant therapy comprised of capecitabine and trastuzumab after the patient gave her informed consent. Therapy comprised of capecitabine and trastuzumab was chosen because her tumors were not resistant to these medications until resection, and trastuzumab has a significant benefit in tumor cell eradication in the event that the remaining micrometastases retain HER2-positivity. Third, clinicians need to know if the loss of HER2 positivity has a significant prognostic impact on MUGC, because some studies have shown that loss of HER2 positivity in HER2-positive breast cancer was associated with a significantly worse prognosis compared to patients with tumors that maintained HER2 expression $[12,18,20,21]$.

Even if a marked response to chemotherapy allows the possibility of curative resection in MUGC, currently, the consistent standard treatment is to continue chemotherapy. However, the survival rate in MUGC treated by chemotherapy alone is obviously insufficient. Surgical resection with curative intent after a response to chemotherapy for MUGC is called adjuvant or salvage surgery. The benefit of such surgery was recently evaluated, and a favorable prognosis was suggested, with a 3-year survival rate of $46-55 \%$ and a median survival of 29-43 months [30-32], although these studies were small and retrospective in nature. In carefully selected patients, adjuvant surgery might become the treatment of choice. To support the efficacy of adjuvant surgery, a randomized controlled study is warranted.

In conclusion, we report the case of a patient with HER2positive MUGC at initial diagnosis who received chemotherapy including trastuzumab; following chemotherapy and surgery in this patient, a resected specimen of the stomach showed loss of HER2-positivity in the residual tumor, suggesting that HER2 status is not always stable. Treatment with trastuzumab is very effective and less toxic than traditional cytotoxic drugs. If HER2 positivity is gained during the course of disease, it is an easy judgment for clinicians to add trastuzumab to chemotherapy, thus allowing patients to benefit from trastuzumab therapy. However, when cases convert from positive HER2 status to negative HER2 status, clinicians remain uncertain as to whether the change in status is truly due to a biologic change rather than a false-negative result. Therefore, it is a more difficult and less confident judgment to remove trastuzumab from chemotherapy, even though its removal may result in avoiding unnecessary treatment. Further studies concerning HER2 status in gastric cancer are needed in order to appropriately use trastuzumab for these patients.

\section{Conflict of Interests}

The authors declare that there is no conflict of interests regarding the publication of this paper.

\section{References}

[1] E. Van Cutsem, V. M. Moiseyenko, S. Tjulandin et al., "Phase III study of docetaxel and cisplatin plus fluorouracil compared with cisplatin and fluorouracil as first-line therapy for advanced gastric cancer: a report of the V325 study group," Journal of Clinical Oncology, vol. 24, no. 31, pp. 4991-4997, 2006.

[2] W. Koizumi, H. Narahara, T. Hara et al., "S-1 plus cisplatin versus S-1 alone for first-line treatment of advanced gastric cancer (SPIRITS trial): a phase III trial," The Lancet Oncology, vol. 9, no. 3, pp. 215-221, 2008.

[3] D. Cunningham, N. Starling, S. Rao et al., "Capecitabine and oxaliplatin for advanced esophagogastric cancer," The New England Journal of Medicine, vol. 358, no. 1, pp. 36-46, 2008.

[4] Y.-J. Bang, E. van Cutsem, A. Feyereislova et al., "Trastuzumab in combination with chemotherapy versus chemotherapy alone 
for treatment of HER2-positive advanced gastric or gastrooesophageal junction cancer (ToGA): a phase 3, open-label, randomised controlled trial," The Lancet, vol. 376, no. 9742, pp. 687-697, 2010.

[5] D. J. Slamon, W. Godolphin, L. A. Jones et al., "Studies of the HER-2/neuproto-oncogene in human breast and ovarian cancer," Science, vol. 244, no. 4905, pp. 707-712, 1989.

[6] D. M. Reese and D. J. Slamon, "HER-2/neu signal transduction in human breast and ovarian cancer," Stem Cells, vol. 15, no. 1, pp. 1-8, 1997.

[7] D. J. Slamon, B. Leyland-Jones, S. Shak et al., "Use of chemotherapy plus a monoclonal antibody against her2 for metastatic breast cancer that overexpresses HER2," The New England Journal of Medicine, vol. 344, no. 11, pp. 783-792, 2001.

[8] L. Gianni, U. Dafni, R. D. Gelber et al., "Treatment with trastuzumab for 1 year after adjuvant chemotherapy in patients with HER2-positive early breast cancer: a 4-year follow-up of a randomised controlled trial," The Lancet Oncology, vol. 12, no. 3, pp. 236-244, 2011.

[9] L. Gianni, W. Eiermann, V. Semiglazov et al., "Neoadjuvant and adjuvant trastuzumab in patients with HER2-positive locally advanced breast cancer (NOAH): follow-up of a randomised controlled superiority trial with a parallel HER2-negative cohort," The Lancet Oncology, vol. 15, no. 6, pp. 640-647, 2014.

[10] J. Liu, H. Deng, W. Jia et al., "Comparison of ER/PR and HER2 statuses in primary and paired liver metastatic sites of breast carcinoma in patients with or without treatment," Journal of Cancer Research and Clinical Oncology, vol. 138, no. 5, pp. 837842, 2012.

[11] R. Nakamura, N. Yamamoto, Y. Onai, Y. Watanabe, H. Kawana, and M. Miyazaki, "Importance of confirming HER2 overexpression of recurrence lesion in breast cancer patients," Breast Cancer, vol. 20, no. 4, pp. 336-341, 2013.

[12] M. V. Dieci, E. Barbieri, F. Piacentini et al., "Discordance in receptor status between primary and recurrent breast cancer has a prognostic impact: a single-institution analysis," Annals of Oncology, vol. 24, no. 1, Article ID mds248, pp. 101-108, 2013.

[13] E. M. de Dueñas, A. L. Hernández, Á. G. Zotano et al., "Prospective evaluation of the conversion rate in the receptor status between primary breast cancer and metastasis: results from the GEICAM 2009-03 ConvertHER study," Breast Cancer Research and Treatment, vol. 143, no. 3, pp. 507-515, 2014.

[14] C. Xiao, Y. Gong, E. Y. Han, A. M. Gonzalez-Angulo, and N. Sneige, "Stability of HER2-positive status in breast carcinoma: a comparison between primary and paired metastatic tumors with regard to the possible impact of intervening trastuzumab treatment," Annals of Oncology, vol. 22, no. 7, pp. 1547-1553, 2011.

[15] T. Shet, A. Agrawal, R. Chinoy, R. Havaldar, V. Parmar, and R. Badwe, "Changes in the tumor grade and biological markers in locally advanced breast cancer after chemotherapyimplications for a pathologist," The Breast Journal, vol. 13, no. 5, pp. 457-464, 2007.

[16] M. Kasami, T. Uematsu, M. Honda et al., "Comparison of estrogen receptor, progesterone receptor and Her-2 status in breast cancer pre- and post-neoadjuvant chemotherapy," Breast, vol. 17, no. 5, pp. 523-527, 2008.

[17] T. Hirata, C. Shimizu, K. Yonemori et al., "Change in the hormone receptor status following administration of neoadjuvant chemotherapy and its impact on the long-term outcome in patients with primary breast cancer," British Journal of Cancer, vol. 101, no. 9, pp. 1529-1536, 2009.
[18] E. A. Mittendorf, Y. Wu, M. Scaltriti et al., "Loss of HER2 amplification following trastuzumab-based neoadjuvant systemic therapy and survival outcomes," Clinical Cancer Research, vol. 15, no. 23, pp. 7381-7388, 2009.

[19] V. Guarneri, M. V. Dieci, E. Barbieri et al., "Loss of HER2 positivity and prognosis after neoadjuvant therapy in HER2positive breast cancer patients," Annals of Oncology, vol. 24, no. 12, pp. 2990-2994, 2013.

[20] N. Niikura, J. Liu, N. Hayashi et al., "Loss of human epidermal growth factor receptor 2 (HER2) expression in metastatic sites of HER2-overexpressing primary breast tumors," Journal of Clinical Oncology, vol. 30, no. 6, pp. 593-599, 2012.

[21] D. Pectasides, A. Gaglia, P. Arapantoni-Dadioti et al., "HER$2 /$ neu status of primary breast cancer and corresponding metastatic sites in patients with advanced breast cancer treated with trastuzumab-based therapy," Anticancer Research, vol. 26, no. 1, pp. 647-654, 2006.

[22] M. Hofmann, O. Stoss, D. Shi et al., "Assessment of a HER2 scoring system for gastric cancer: results from a validation study," Histopathology, vol. 52, no. 7, pp. 797-805, 2008.

[23] J. Rüschoff, W. Hanna, M. Bilous et al., "HER2 testing in gastric cancer: a practical approach," Modern Pathology, vol. 25, no. 5, pp. 637-650, 2012.

[24] A. H. Marx, L. Tharun, J. Muth et al., "HER-2 amplification is highly homogenous in gastric cancer," Human Pathology, vol. 40, no. 6, pp. 769-777, 2009.

[25] C. Bozzetti, F. V. Negri, C. A. Lagrasta et al., "Comparison of HER2 status in primary and paired metastatic sites of gastric carcinoma," British Journal of Cancer, vol. 104, no. 9, pp. 13721376, 2011.

[26] F. Pagni, S. Zannella, S. Ronchi, C. Garanzini, and B. E. Leone, "HER2 status of gastric carcinoma and corresponding lymph node metastasis," Pathology and Oncology Research, vol. 19, no. 1, pp. 103-109, 2013.

[27] N. Ikari, G. Nakajima, K. Taniguchi et al., "HER2-positive gastric cancer with paraaortic nodal metastasis successfully resected after chemotherapy with trastuzumab: a case report," Anticancer research, vol. 34, no. 2, pp. 867-872, 2014.

[28] H. Yoshida, N. Yamamoto, H. Taniguchi et al., "Comparison of HER2 status between surgically resected specimens and matched biopsy specimens of gastric intestinal-type adenocarcinoma," Virchows Archiv, vol. 465, no. 2, pp. 145-154, 2014.

[29] National Comprehensive Cancer Network, NCCN Clinical Practice Guidelines in Oncology: Breast Cancer, Version 3, 2014.

[30] H. Okabe, S. Ueda, K. Obama, H. Hosogi, and Y. Sakai, "Induction chemotherapy with S-1 plus cisplatin followed by surgery for treatment of gastric cancer with peritoneal dissemination," Annals of Surgical Oncology, vol. 16, no. 12, pp. 3227-3236, 2009.

[31] T. Suzuki, K. Tanabe, J. Taomoto et al., "Preliminary trial of adjuvant surgery for advanced gastric cancer," Oncology Letters, vol. 1, no. 4, pp. 743-747, 2010.

[32] T. Kanda, K. Yajima, S.-I. Kosugi, T. Ishikawa, Y. Ajioka, and K. Hatakeyama, "Gastrectomy as a secondary surgery for stage IV gastric cancer patients who underwent S-1-based chemotherapy: a multi-institute retrospective study," Gastric Cancer, vol. 15, no. 3, pp. 235-244, 2012. 


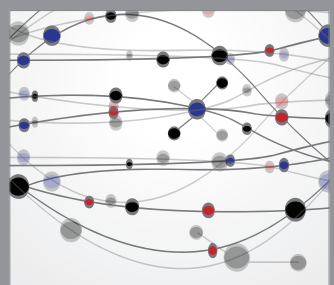

The Scientific World Journal
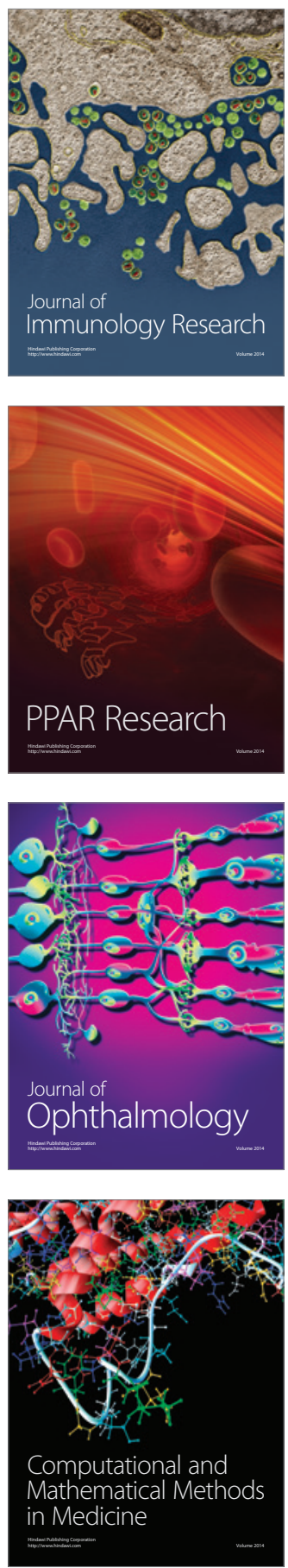

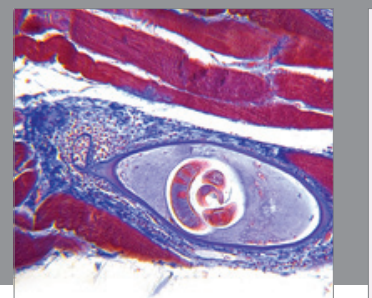

Gastroenterology

Research and Practice
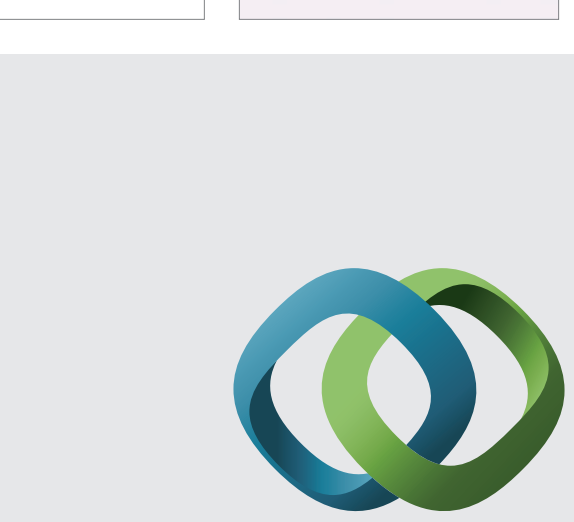

\section{Hindawi}

Submit your manuscripts at

http://www.hindawi.com
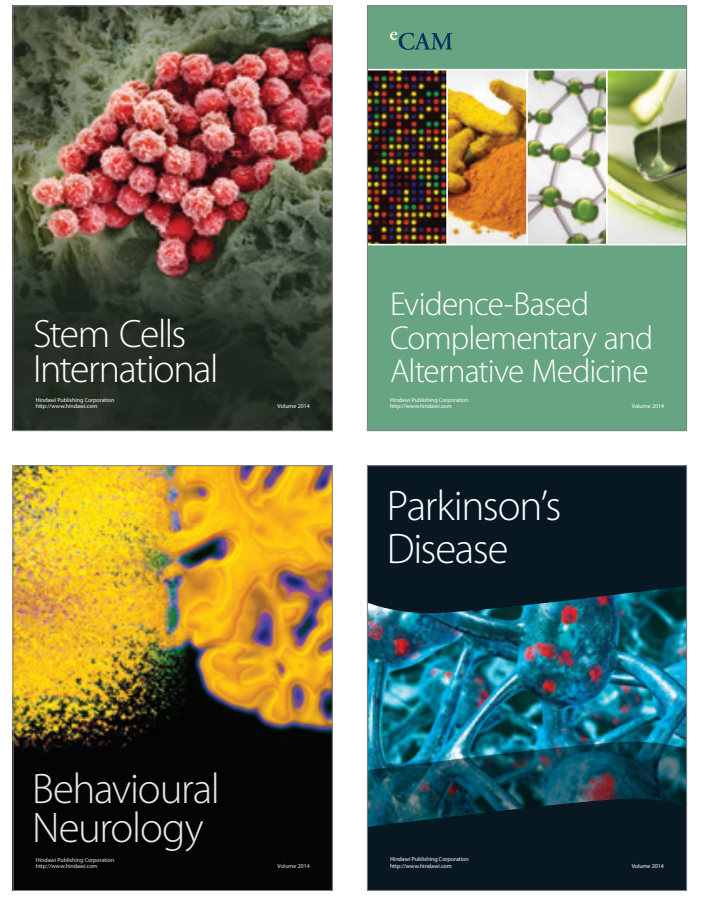
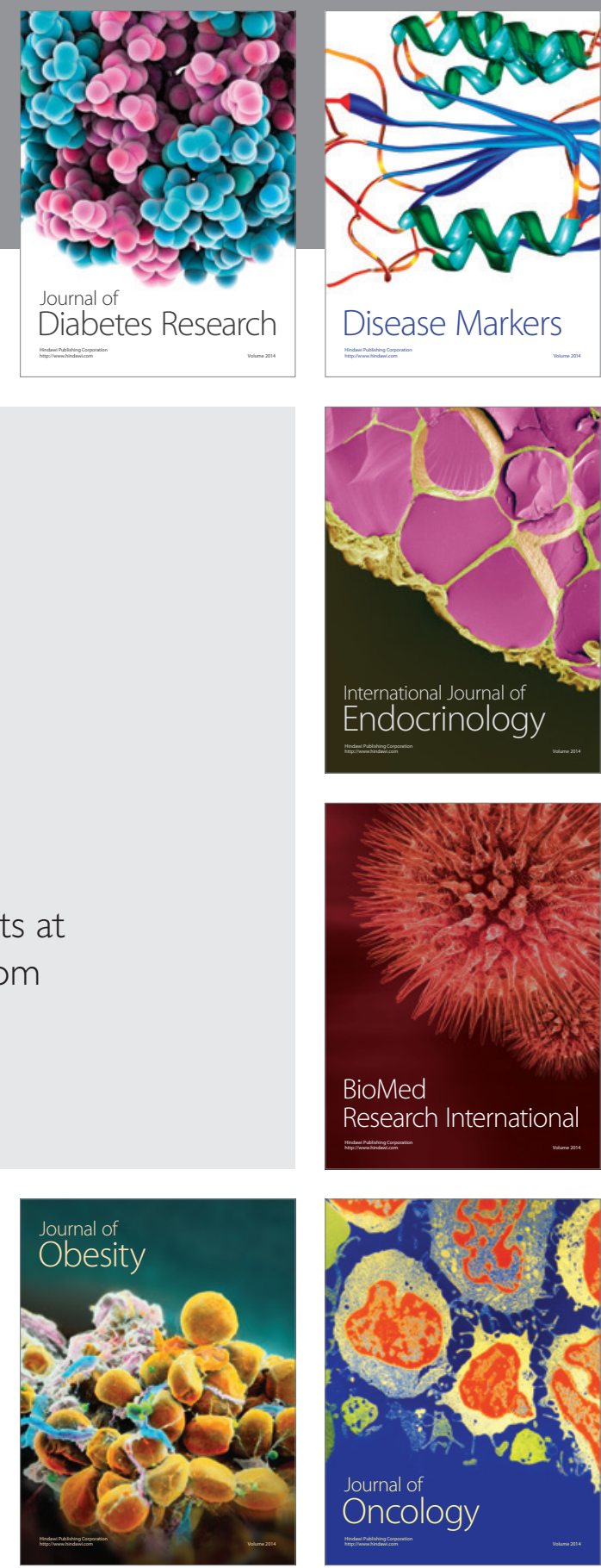

Disease Markers
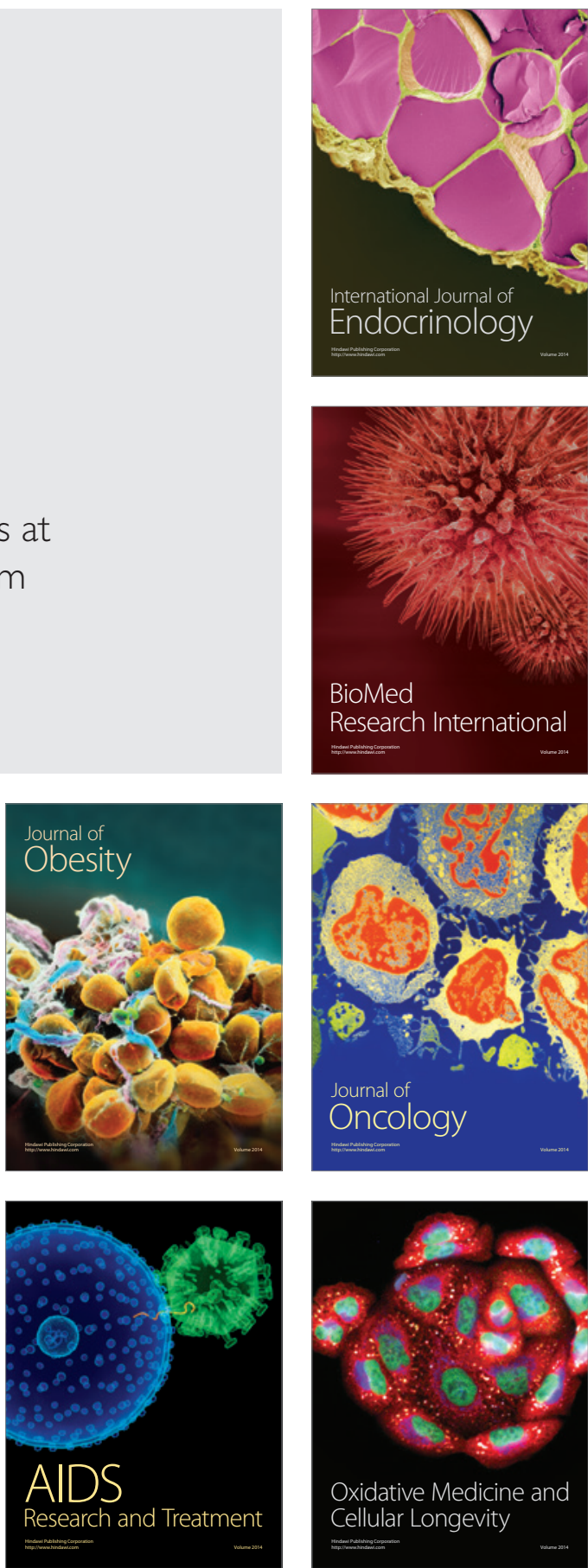